REPORTE DE CASO

\title{
Manifestaciones tóxicas cardiovasculares con el uso de antimoniales en una paciente con leishmaniasis cutánea
}

Fecha de recibido: 16 de mayo de 2018

Fecha de aprobación: 22 de agosto de 2018
Forma de citar este artículo: Valverde A, Berrouet MC. Manifestaciones tóxicas cardiovasculares con el uso de antimoniales en una paciente con leishmaniasis cutánea. Med UPB. 2019;38(1):78-84.

DOI:10.18566/medupb.v38n1.a10

1. Residente de Medicina de Urgencias. Universidad CES. Medellín, Colombia.

2. Toxicóloga clínica. Hospital General de Medellín. Medellín, Colombia.

Dirección de correspondencia: Adrián Valverde Legarda. Correo electrónico: avalverdelegarda@gmail. com
Cardiovascular toxic manifestations with the use of antimonials in a patient with cutaneous leishmaniasis / Manifestações tóxicas cardiovasculares com o uso de antimoniais em uma paciente com leishmanioses cutânea

Adrián Valverde Legarda ${ }^{1}$, Marie Claire Berrouet ${ }^{2}$

\section{RESUMEN}

La toxicidad por antimoniales tiene múltiples manifestaciones sistémicas, entre ellas, las alteraciones cardiovasculares son de las de mayor gravedad, que pueden, incluso, llevar a la muerte. Reportamos el caso de una paciente anciana que durante el tratamiento con antimoniales para una leishmaniasis cutánea presentó toxicidad cardiaca y hepática con alteraciones electrolíticas como factor perpetuador de su condición clínica y con posterior desarrollo de múltiples episodios de taquicardia ventricular monomórfica y polimórfica. Durante el tratamiento recibió descargas sincrónicas y asincrónicas del desfibrilador, sobreestimulación con marcapasos y reposición de electrolitos, no se logró resolver del cuadro y la paciente falleció. Este reporte sobre la toxicidad de los antimoniales es importante porque están en la primera línea en el manejo de la leishmaniasis, a pesar de sus efectos sobre múltiples sistemas, por tanto, es necesario que los trabajadores de la salud se familiaricen con los signos y síntomas tempranos de su toxicidad.

Palabras clave: toxicidad; leishmania; arritmias cardiacas.

\section{ABSTRACT}

Toxicity by antimonials has multiple systemic manifestations, including cardiovascular alteration, which is one of the most serious, as it can even lead to death of the patient. This article reports the case of an elderly patient who, during treatment with antimonials for cutaneous leishmaniasis, presented cardiac and hepatic toxicity associated with electrolyte imbalance as a perpetuating factor of her clinical condition, with subsequent development of multiple episodes of mono and polymorphic ventricular tachycardia. The patient was treated with synchronous and asynchronous defibrillation, overstimulation with pacemaker, and electrolyte replacement; she did not recover and eventually died. This report on the toxicity of antimonials is important as they remain the first-line treatment for leishmaniasis despite their multiple side effects, which can compromise multiple systems. Thus, health care professionals must become familiar with the early signs and symptoms of toxicity.

Key words: toxicity; leishmania; arrhythmias, cardiac.

\section{RESUMO}

A toxicidade por antimoniais tem múltiplas manifestações sistémicas, entre elas, as alterações cardiovasculares são das de maior gravidade, que podem, inclusive, levar à morte. Reportamos o caso de uma paciente anciã que durante o tratamento com antimoniais para uma leishmaniose cutânea apresentou toxicidade cardíaca e hepática com alterações eletrolíticas como fator perpetuador de sua condição clínica e com 
posterior desenvolvimento de múltiplos episódios de taquicardia ventricular monomórfica e polimórfica. Durante o tratamento recebeu descargas sincrónicas e asincrónicas do desfibrilador, sobre estimulação com marca-passos e reposição de eletrólitos, não conseguiu resolver o quadro e a paciente faleceu. Este relatório sobre a toxicidade dos antimoniais é importante porque estão na primeira linha no manejo da leishmaniose, apesar dos seus efeitos sobre múltiplos sistemas, por tanto, é necessário que os trabalhadores da saúde se familiarizem com os signos e sintomas precoces da sua toxicidade. Palavras chave: toxicidade; leishmania; arritmias cardíacas.

\section{INTRODUCCIÓN}

La leishmaniasis es una zoonosis y un problema de salud pública en Colombia, pues hay zonas endémicas distribuidas a lo largo del país ${ }^{1}$. Entre sus presentaciones, la forma cutánea cuenta con reportes epidemiológicos locales ${ }^{2}$. En Colombia y Latinoamérica, el tratamiento con antimoniales pentavalentes es de primera elección para las diversas formas clínicas de la enfermedad. Si bien la mayoría de los eventos adversos de este tratamiento son leves o moderados, los efectos cardiacos se presentan hasta en un $8 \%$ de los pacientes, aun con dosis terapéuticas ${ }^{3}$. Presentamos el caso de una paciente con leishmaniasis cutánea que desarrolló toxicidad cardiaca por antimoniato de meglumina, con un síndrome de QT largo, adquirido con complicaciones como taquicardias ventriculares monomórficas y polimórficas.

\section{CASO CLÍNICO}

Paciente femenina de 77 años, procedente de zona rural. Con cuadro de dos meses de evolución que empezó con una lesión en el antebrazo izquierdo. Recibió tratamiento antibiótico (se desconoce cuál), con inicio subsecuente de diarrea. Sumado a lo anterior, presenta hipertensión en manejo con losartan e hidroclorotiazida.

Se le diagnosticó leishmaniasis cutánea y le inician Glucantime ${ }^{\circledR}$, que recibe por 17 días. En el transcurso del tratamiento, la paciente continúa con diarrea y adicionalmente se evidenciaron lesiones en glúteo izquierdo, sitio de aplicación del medicamento.

Fue hospitalizada en un centro de mediana complejidad donde se identificó hipocalemia moderada $(2.7 \mathrm{meq} / \mathrm{L})$. Se dio el alta y reingresó a las 24 horas con hipocalemia grave $(2.25 \mathrm{meq} / \mathrm{L})$. Se inició reposición de potasio por vía intravenosa. En el seguimiento tuvo un episodio de taquicardia ventricular monomórfica, manejada con amiodarona $(1 \mathrm{mg} / \mathrm{min}$ y luego $0.5 \mathrm{mg} / \mathrm{min}$ ), suspendida por hipotensión sintomática. Dado que no mejoraba, se decide el traslado a una institución de alta complejidad.

$\mathrm{Al}$ ingreso al nivel 3 de atención, fue documentado un episodio de taquicardia ventricular (TV) polimórfica, manejada con terapia eléctrica 100 Joules.

Durante el primer día de observación se diagnosticó taquicardia de puntas torcidas (Figura 1) y se inició sulfato de magnesio, con buena respuesta. La paciente fue monitorizada y se lograron documentar extrasístoles ventriculares y prolongación del intervalo QT (Figura 2). Adicionalmente se identificó una crisis hiperglicémica por lo que se aplicó insulina venosa. También se detectó elevación de transaminasas, que es indicativa de hepatotoxicidad. En la Tabla 1 se presentan varios paraclínicos realizados en diversas fechas a la paciente.

Después, presentó más episodios de taquicardia de puntas torcidas, de menos de 10 segundos de duración, por lo que se trató nuevamente con sulfato de magnesio. Cinco horas después presentó un nuevo episodio de TV polimórfica (Figura 3), que requirió cardioversión con 200 Joules. En ese momento se decidió poner marcapasos transvenoso. Durante el procedimiento la paciente tuvo un nuevo episodio de taquicardia polimórfica y fue cardiovertida con 150 Joules.

Al día siguiente la paciente seguía con el marcapasos y, a pesar de esto, presentó paro cardiorrespiratorio y fibrilación ventricular documentada, que cedió luego 


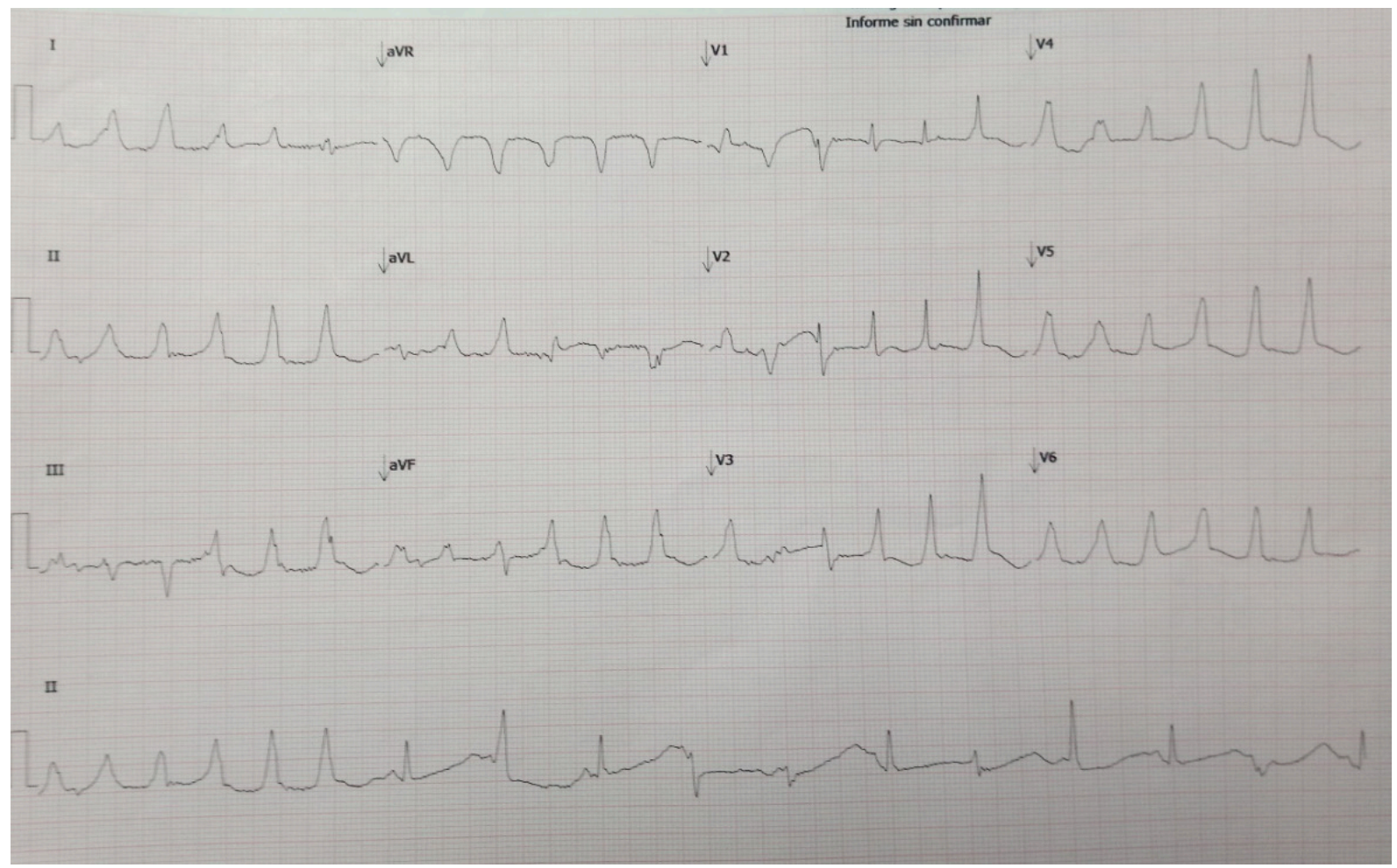

Figura 1. Taquicardia de puntas torcidas

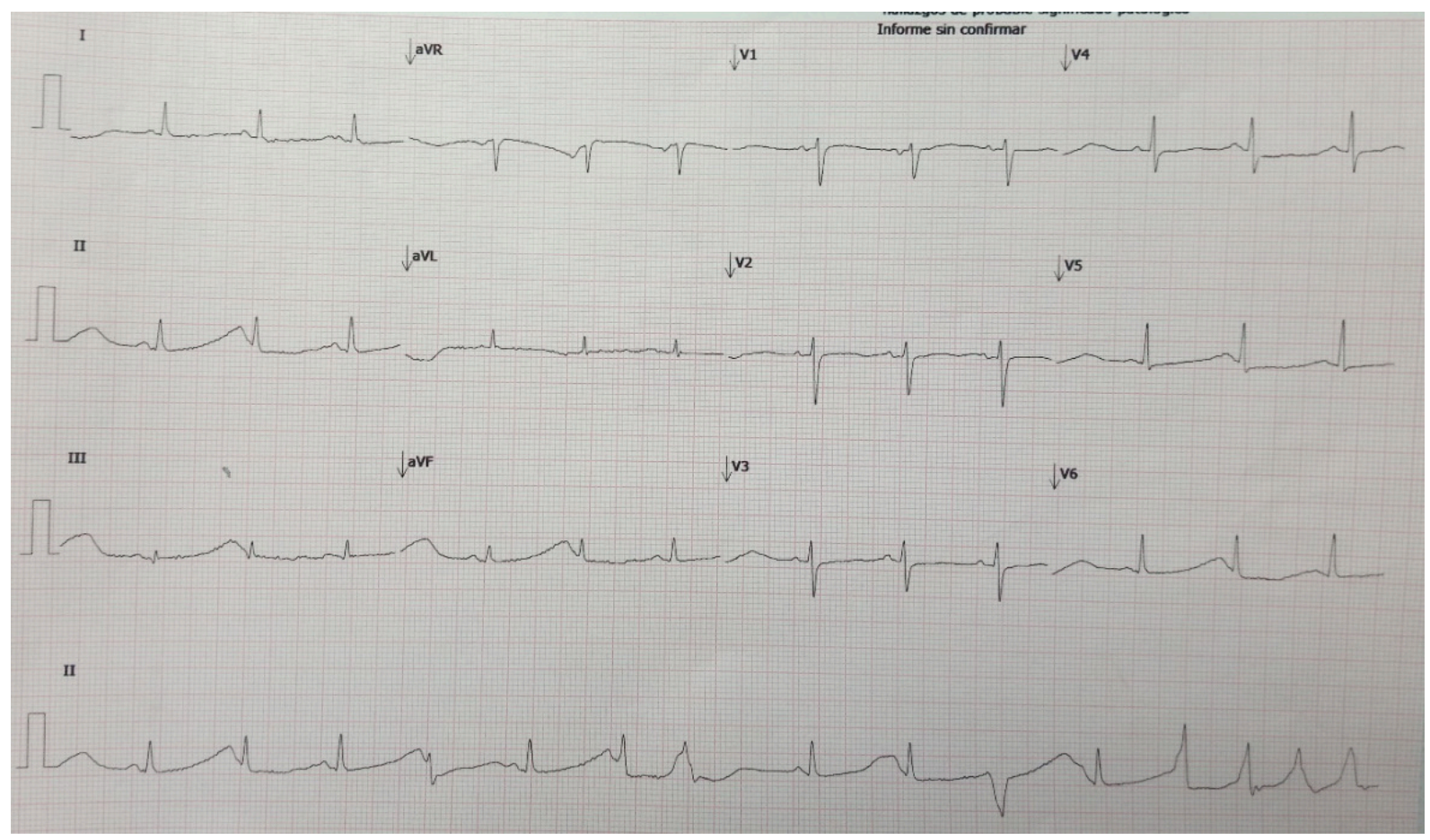

Figura 2. Prolongación del intervalo QT 
Tabla 1. Paraclínicos y fecha de realización.

\begin{tabular}{|c|c|c|c|c|c|c|}
\hline Examen/2018 & 26.04 & 27.04 & 28.04 & 28.04 & 29.04 & 30.04 \\
\hline Leucocitos & 4200 & & & 8500 & & \\
\hline PMN (\%) & 62 & & & 80 & & \\
\hline Linfocitos (\%) & 18 & & & 8.5 & & \\
\hline $\mathrm{Hb}(\mathrm{g} / \mathrm{dl})$ & 11 & & 10.2 & 10 & & 10.2 \\
\hline Hto (\%) & 32.8 & & 30 & 31.1 & & \\
\hline Plaquetas & 369000 & & & & & \\
\hline Sodio (mEq/L) & 141 & 142 & 147 & 147 & 152 & 146 \\
\hline Potasio(mEq/L) & 2.25 & 2.75 & 2.3 & 3.05 & 2.89 & 3.16 \\
\hline Cloro(mEq/L) & 101 & 100 & & 103 & & \\
\hline Calcio & 1.06 & 1 & & 7.3 & & \\
\hline Fósforo & & & & 2.32 & & \\
\hline Magnesio & & & & 2.51 & 2.76 & 2.54 \\
\hline Creatinina & & & & 1.32 & 1.24 & \\
\hline $\mathrm{pH}$ & & 7.48 & 7.46 & & & 7.16 \\
\hline PCO2 & & 33.2 & 35 & & & 36 \\
\hline PO2 & & 63.2 & 69 & & & \\
\hline $\mathrm{HCO} 3$ & & 24.3 & 25.4 & & & 13 \\
\hline $\mathrm{BE}$ & & & 2 & & & -15 \\
\hline Glucometría (mg/dl) & & & 346 & 356 & & \\
\hline TP/INR & & & & 16.6/1.15 & & \\
\hline TPT & & & & 35.2 & & \\
\hline AST & & & & 386 & & \\
\hline ALT & & & & 168 & & \\
\hline Bilirrubina Total/directa & & & & $1.1 / 1.1$ & & \\
\hline Troponina I & & $<0.1$ & & $<0.012$ & & \\
\hline Cuerpos cetónicos & & & & 0.8 & & \\
\hline
\end{tabular}

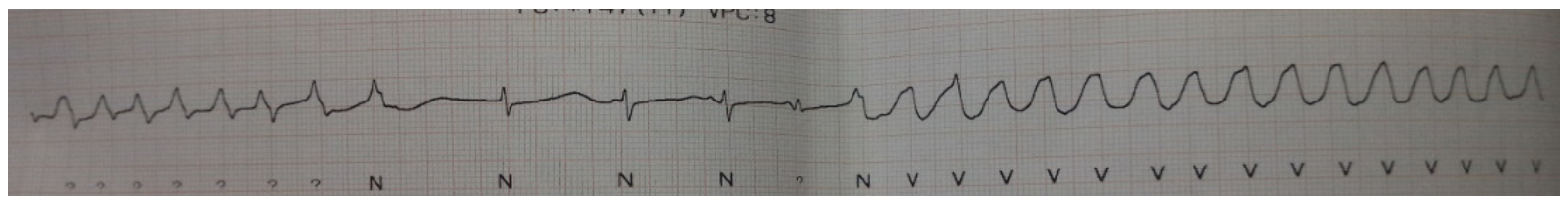

Figura 3. Taquicardia ventricular polimórfica

de dos ciclos de masaje cardiaco y de descarga de 200 Joules. Se inició soporte vasopresor con norepinefrina. Posteriormente fue valorada por un especialista en electrofisiología quien mantuvo el plan de sobreestimulación con marcapasos y estuvo de acuerdo con la propuesta del área de cardiología, de tratar con isoproterenol.

Dos días después del ingreso, la paciente continuaba con mala respuesta hemodinámica, por lo que se hizo necesario aumentar el vasopresor. Fue valorada por toxicología que decidió seguir el soporte ante la ausencia de terapia específica. Se clasificó la reacción como tipo
$\mathrm{B}$, idiosincrática, no prevenible. También se aclaró que la hipocalemia es un factor de riesgo para prolongación adquirida del intervalo QT. La paciente presentó nuevo episodio de paro cardiorrespiratorio, que no se pudo recuperar con las maniobras realizadas y falleció.

\section{DISCUSIÓN}

Los compuestos antimoniales pentavalentes como el antimoniato de meglumina (Glucantime ${ }^{\circledR}$ ) y el estibogluconato de sodio $\left(\right.$ Pentostam $\left.^{\circledR}\right)$ continúan siendo 
utilizados en el manejo de la leishmaniasis tanto en humanos como en animales, y es una de las primeras líneas terapéuticas a pesar de sus efectos adversos. Si bien es cierto que hay reportes del uso de otros medicamentos, como la anfotericina $\mathrm{B}{ }^{4,5}$, tambien es cierto que en nuestra región los antimoniales siguen siendo de elección, con evidencia de buena calidad ${ }^{3,6}$.

Aunque su mecanismo de acción no está claro, diferentes autores han plateado que los antimoniales alteran procesos como la glucolisis y el metabolismo de ácidos grasos del parásito, lo que lleva, en última instancia, a una depleción energética ${ }^{3,6}$.

Dentro de las características cinéticas de los antimoniales pentavalentes, autores como Gamboa, proponen que tienen dos fases de eliminación, la segunda es más duradera (entre 33 y 72 horas). En esta fase extendida se plantea la posibilidad de biotransformación a formas trivalentes más tóxicas ${ }^{7}$.

Desde los modelos animales hasta los reportes de caso, se han descrito grados variables de toxicidad ${ }^{8}$. Entre los efectos adversos se han descrito: mialgias, artralgias, hepatotoxicidad con patrón colestásico, toxicidad ótica, compromiso hematológico (leucopenia), toxicodermias, cardiotoxicidad, alteraciones renales y pancreatitis. Algunos autores reportan que la frecuencia de efectos adversos es hasta del $21 \%{ }^{8,9}$.

Aunque algunos modelos en animales no encuentran cambios electrocardiográficos significativos ${ }^{10}$, sí existe evidencia de toxicidad cardiaca en humanos. Dentro de los cambios electrocardiográficos reportados están: anormalidades en la onda $T$, taquicardias ventriculares, taquicardia de puntas torcidas y prolongación del intervalo $\mathrm{QT}^{11}$.

Sundar et al., en 1998 reportaron el seguimiento a 130 pacientes realizado durante un año en el que hubo una frecuencia de cardiotoxicidad menor al 10\%. Dentro de los factores asociados a la misma se resaltan el cambio de lote del medicamento y el tiempo transcurrido, ya que en los casos se presentaron entre tres y ocho días. Dentro de las alteraciones reportadas estuvieron la falla cardiaca y la prolongación del QT. En esta descripción se informaron seis muertes, las dosis acumulativas fueron menores a $300 \mathrm{mg} / \mathrm{kg}$ y algunas de ellas fueron relacionadas con taquicardia ventricular, puntas torcidas y fibrilación ventricular. La toxicidad fue reportada con dosis desde $15 \mathrm{mg} / \mathrm{kg}^{11,12}$.

Diez años más tarde, Sadeghian et al., conocedores del riesgo de cardiotoxicidad, realizaron un estudio descriptivo para evaluar cambios electrocardiográficos en el que la alteración más frecuente, después del mes de seguimiento, fue la prolongación del QT (en el 19\%), la segunda fue la alteración de la onda $\mathrm{T}(7.4 \%)$ y en un 6.1\% de los sujetos, las alteraciones del segmento $\mathrm{ST}^{13}$.
Hallazgos similares fueron reportados en el 2013 por Shanehsaz et $a l^{14}$.

$\mathrm{Si}$ bien los autores que han estudiado este tema resaltan que se trata de una reacción tipo B de la clasificación de Aronson (es decir, idiosincrática, independiente de la dosis, de la edad y el género ${ }^{15}$, coinciden en reportar el QT prolongado y las arritmias ventriculares como uno los hallazgos más frecuentes. También llaman la atención sobre la susceptibilidad de los individuos con QT adquirido ${ }^{7,16}$.

La importancia de la prolongación congénita o adquirida del intervalo QT radica en que en esta anomalía esta alterada la repolarización, lo que puede llevar a ritmos ventriculares y a muerte súbita ${ }^{14,17}$. E1 QT largo congénito es una canalopatía descrita por Jervell y Lange-Nielsen en 1957. En ella hay riesgo de síncope, taquicardia ventricular polimórfica y muerte súbita. Algunos calculan su prevalencia en 1 por cada 2000 nacidos. Las mutaciones relacionadas frecuentemente con esta condición son la SQTL1, (canales lentos de potasio Iks), la SQTL2 (canales rápidos de potasio IKr) y la SQTL3 (canales de sodio Nav1) ${ }^{14}$. En esta última forma los eventos cardiacos son menos frecuentes pero más letales ${ }^{18}$.

Con respecto al QT largo adquirido, es importante resaltar que esta alteración es mucho más frecuente que la congénita. Reportada desde 1920, ha sido motivo de retiro del mercado de varios medicamentos ${ }^{17}$. Muchos clínicos, al prescribir, asocian este riesgo solo a familias específicas de psicofármacos o antiarrítmicos, sin embargo, la lista es más amplia, de ahí la importancia de consultar bases de datos de seguridad de medicamentos e identificar otros posibles factores de riesgo en pacientes particulares. Entre dichos factores de riesgo se cuentan las alteraciones hidroelectrolíticas, específicamente la hipocalemia y la hipomagnesemia. Además, la edad avanzada, el sexo femenino, la disfunción hepática o renal aumentan el riesgo de arritmias, incluida la taquicardia ventricular polimórfica ${ }^{17}$. Una de las explicaciones implica el uso de medicamentos que interactúan con el canal de potasio codificado por genes HERG ${ }^{19,20}$.

En el presente caso se observan los criterios de causalidad de Bradford Hill: temporalidad, plausibilidad biológica, coherencia y congruencia ${ }^{21}$. Al igual que en el reporte de caso de Gamboa, en donde el antimonial utilizado fue estibogluconato de sodio, también se evidenció el compromiso multisistémico (renal, hepático y cardiovascular) así como un sinergismo entre el QT prolongado y la hipocalemia grave, para el desarrollo de arritmias ventriculares ${ }^{7}$. Más aún, el algoritmo de Naranjo para evaluar la causalidad en una reacción medicamentosa tuvo un puntaje de seis, que indica reacción adversa en el rango de probable ${ }^{22}$. 
En conclusión, el uso de medicamentos antimoniales seguirá siendo una de las primeras líneas de tratamiento para la leishamaniasis, a pesar de que los efectos secundarios pueden llegar a ser graves y letales. La paciente reportada presentó disfunción orgánica múltiple en la que resalta el compromiso electrolítico, hepático y cardiovascular, este último evidenciado en los episodios de TV polimorfa. Dado que en el contexto del uso de estos medicamentos está reportada la prolongación del QT, llama la atención la hipocalemia en esta paciente cuya causa también se vio condicionada por la presencia de diarrea. A su vez, la hipocalcemia constituye un factor predisponente para arritmias y se asocia al uso de amiodarona, que por su mecanismo de acción incide sobre la prolongación del QT.
Entendiendo que no hay un tratamiento específico de esta toxicidad, es importante individualizar el manejo de los pacientes y hacer un seguimiento riguroso de factores de riesgo antes de iniciar antimoniales. Al momento de detectar signos y síntomas inespecíficos, hay que tener la precaución de sospechar tempranamente que la medicación puede estar produciendo manifestación de toxicidad y, en tal caso, suspenderla y realizar un abordaje oportuno.

\section{DECLARACIÓN DE CONFLICTO DE INTERESES}

Los autores declaran no tener ningún conflicto de intereses.

\section{REFERENCIAS}

1. Vélez D, Hendrickx E, Robledo SM, Agudelo SP. Leishmaniosis cutánea en Colombia y género. Cad Saúde Pública 2001; 17(1):171-180.

2. Gaitán H, Lasso G, Gaitán H. Leishmaniasis cutánea en la provincia del Magdalena Centro, Cundinamarca, Colombia. Una serie de casos. Universitas Medica 2017; 58(2):2015-2018.

3. Santamaría M, Soto B, Velásquez I, Casas J, Castaño V, Benito G, et al. Guía para la atención clínica integral del paciente con leishmaniasis. Minsalud 2010;129.

4. Nicolas G, Koury E, Osman D, Salibi C, Nehme L, Mitri S, et al. Leishmania in a patient with small lymphocytic lymphoma/chronic lymphocytic leukemia. Am J Case Rep 2018; 19:512516.

5. Vélez JD, Carrillo DC. Leishmaniasis cutánea y anfotericina B liposomal. Reporte de caso. Infectio 2013; 17(4):201-204.

6. OPS. Leishmaniasis en las Américas: recomendaciones para el tratamiento. 2013. 1-46 p.

7. Gamboa LF, Becerra JA. Cardiotoxicidad por antimoniales. Rev Salud Bosque. 2012; 2(2):69-74.

8. Henao HH, Osorio Y, Saravia NG, Gómez A, Travi B. Eficacia y toxicidad de los antimoniales pentavalentes (Glucantime y Pentostam) en un modelo animal de leishmaniasis cutánea americana: aplicación de la luminometría. Biomédica 2004; 24(4):393.

9. Masmoudi A, Maalej N, Mseddi M, Souissi A, Turki H, Boudaya S, et al. Glucantime ${ }^{\circledR}$ par voie parentérale : bénéfice versus toxicité. Médecine Mal Infect 2005; 35(1):42-45.

10. Luciani A, Sconza S, Civitella C, Guglielmini C. Evaluation of the cardiac toxicity of N-methylglucamine antimoniate in dogs with naturally occurring leishmaniasis. Vet J 2013; 196(1):119-121.

11. Matoussi N, Ameur HB, Amor SB, Fitouri Z, Becher SB. Toxicité cardiaque de l'antimoniate de méglumine (Glucantime ${ }^{\circledR}$ ). À propos d'une observation. Med Mal Infect 2007; 37(SUPPL. 3):257-259.

12. Sundar S, Sinha PR, Agrawal NK, Srivastava R, Rainey PM, Berman JD, et al. A cluster of cases of severe cardiotoxicity among kala-azar patients treated with a high-osmolarity lot of sodium antimony gluconate. Am J Trop Med Hyg 1998; 59(1):139-143.

13. Sadeghian G, Ziaei $H$, Sadeghi M. Electrocardiographic changes in patients with cutaneous leishmaniasis treated with systemic glucantime. Ann Acad Med Singapore 2008; 37(11):916-918.

14. Arcos L, Rincón C, Vanegas D, Medina R. Tormenta eléctrica y torsade de pointes asociados a tratamiento con antimoniales en un paciente con leishmaniasis cutánea. Rev Colomb Cardiol 2018; 9:5-9.

15. Edwards IR, Aronson JK. Adverse drug reactions Adverse drug reactions: definitions, diagnosis, and management. Lancet 2000; 356:1255-1259.

16. Baranwal AK, Mandal RN, Singh R, Singhi SC. Sodium stibogluconate and polymorphic ventricular tachycardia. Indian J Pediatr 2005; 72(3):269.

17. Villamañán $E$, Armada $E$, Ruano M. Prolongación del intervalo QT inducido por fármacos: ¿conocemos sus riesgos? Med Clin (Barc) 2015; 144(6):269-274.

18. Pérez-Riera AR, Barbosa-Barros R, Daminello R, da Costa de Rezende Barbosa MP, Esposito IC, de Abreu LC. The congenital long QT syndrome Type 3: An update. Indian Pacing Electrophysiol J 2018; 18(1):25-35.

19. Cay S, Ozeke O, Ozcan F, Topaloglu S, Aras D. Long QT: A syndrome or acquired finding? Pacing Clin Electrophysiol 2018; doi: 10.1111/pace.13357.

20. El-Sherif N, Turitto G, Boutjdir M. Acquired long QT syndrome and torsade de pointes. Pacing Clin Electrophysiol 2018; 41(4):414-421. 
REPORTE DE CASO - CASE REPORT - REPORTE DE CASO | Manifestaciones tóxicas cardiovasculares con el uso de antimoniales en una paciente con leishmaniasis cutánea

21. Álvarez HPE. Causalidad en medicina. Gac Med Mex 2004; 140(4):467-472.

22. Invima. Boletín de farmacovigilancia [Internet]. La causalidad en farmacovigilancia. 2004. p. 1-10. Available from: https://www.invima.gov.co/images/stories/boletines/Boletin_5.pdf 